PROCEEDINGS OF THE

AMERICAN MATHEMATICAL SOCIETY

Volume 131, Number 3, Pages 871-877

S 0002-9939(02)06576-0

Article electronically published on August 19, 2002

\title{
NUMERICAL INDEX AND RENORMING
}

\author{
CATHERINE FINET, MIGUEL MARTÍN, AND RAFAEL PAYÁ \\ (Communicated by N. Tomczak-Jaegermann)
}

\begin{abstract}
We study the numerical index of a Banach space from the isomorphic point of view, that is, we investigate the values of the numerical index which can be obtained by renorming the space. The set of these values is always an interval which contains $\left[0,1 / 3\right.$ [ in the real case and $\left[e^{-1}, 1 / 2[\right.$ in the complex case. Moreover, for "most" Banach spaces the least upper bound of this interval is as large as possible, namely 1 .
\end{abstract}

\section{INTRODUCTION}

The numerical index of a Banach space is a constant relating the norm and the numerical radius of operators on the space. For a Banach space $X$, we write $X^{*}$ for the dual space, and $L(X)$ for the Banach algebra of all bounded linear operators on $X$. The numerical radius of such an operator $T$ is given by

$$
v(T)=\sup \left\{\left|x^{*}(T x)\right|: x^{*} \in X^{*}, x \in X, x^{*}(x)=\left\|x^{*}\right\|=\|x\|=1\right\} .
$$

It is clear that $v$ is a seminorm on $L(X)$, and $v(T) \leq\|T\|$ for every $T \in L(X)$. The numerical index of the space $X$ is the constant $n(X)$ defined by

$$
n(X)=\inf \{v(T): T \in L(X),\|T\|=1\}
$$

or, equivalently, $n(X)$ is the greatest constant $k \geq 0$ such that $k\|T\| \leq v(T)$ for every $T \in L(X)$. Note that $0 \leq n(X) \leq 1$, and $n(X)>0$ if and only if $v$ and $\|\cdot\|$ are equivalent norms (the numerical radius can be a non-equivalent norm on $L(X)$; see [13]). Clearly, $n(X)=1$ when the operator norm and the numerical radius agree on $L(X)$. This is the case of some classical spaces such as $L_{1}(\mu)$ and $C(K)$. For general information and background we refer to the books by F. Bonsall and J. Duncan [3, 4]. More recent results can be found in [11, 12, 13] and the references therein.

Let us mention some facts concerning the numerical index which will be relevant to our discussion. For instance, one has $v\left(T^{*}\right)=v(T)$ for every $T \in L(X)$, where $T^{*}$ is the adjoint operator of $T$ (see $[3, \S 9]$ ), and it clearly follows that $n\left(X^{*}\right) \leq n(X)$ for every Banach space $X$. The question of whether this is actually an equality seems to be open. The range of values of the numerical index was described in [6];

Received by the editors May 29, 2001 and, in revised form, October 18, 2001.

2000 Mathematics Subject Classification. Primary 46B20, 47A12.

Key words and phrases. Numerical range, numerical index, renorming.

The first author was partially supported by La Banque Nationale de Belgique.

The second and third authors were partially supported by Spanish MCYT project no. BFM2000-1467. 
for real Banach spaces the numerical index can be any number in the interval $[0,1]$, while for complex spaces the numerical index covers the interval $\left[e^{-1}, 1\right]$. The fact that $n(X) \geq e^{-1}$ for complex $X$ goes back to H. F. Bohnenblust and S. Karlin [2] (see also [8]).

In [11 the authors studied isomorphic properties of real Banach spaces with numerical index 1 . They proved that not every Banach space admits an equivalent norm with numerical index 1. For example, this happens with infinite-dimensional reflexive or quasi-reflexive real Banach spaces. Our aim is to show that, in this context, 1 is a very particular value for the numerical index. To explain our results, let us fix some notations. Given two Banach spaces $X$ and $Y$, we write $X \simeq Y$ when the spaces are (topologically) isomorphic. The set of values of the numerical index that a Banach space $X$ can have up to renorming is then given by

$$
\mathcal{N}(X)=\{n(Y): Y \simeq X\} .
$$

Of course, the one-dimensional space has numerical index 1 . In the sequel, we will deal only with Banach spaces of dimension greater than one.

In 19, T. Tillekeratne proved that $e^{-1} \in \mathcal{N}(X)$ for every complex Banach space $X$, and asked for what he called the "upper topological numerical index" of $X$, namely $\sup \mathcal{N}(X)$. We first observe that $\mathcal{N}(X)$ is always an interval, a fact which is essentially contained in [4]. In our main results we use two geometrical properties, the so-called properties $(\alpha)$ and $(\beta)$ introduced in the study of norm attaining operators (see [10, 17, 18]). We get an estimate of the numerical index of a Banach space with any of these properties in terms of the constant involved in the definitions. Following the proof of Partington's renorming theorem [17] we then get that $\mathcal{N}(X)$ contains $\left[0,1 / 3\left[\right.\right.$ in the real case and $\left[e^{-1}, 1 / 2[\right.$ in the complex case. On the other hand, renorming theorems on property $(\alpha)[9,18$, give us that for a Banach space $X$ with a "long biorthogonal system" $\mathcal{N}(X)$ contains $[0,1[$ in the real case and $\left[e^{-1}, 1[\right.$ in the complex case. Examples of such Banach spaces are weakly compactly generated spaces. So, for this class of Banach spaces, Tillekeratne's upper topological numerical index is always 1.

\section{The Results}

We first point out that every Banach space can be renormed to achieve the minimum possible value of the numerical index. In the complex case this was shown in [19, Theorem 3.1] and the proof can easily be adapted to the real case. For the sake of completeness, we give a short proof.

Proposition 1. Let $X$ be a Banach space of dimension greater than one. Then $0 \in \mathcal{N}(X)$ in the real case, and $e^{-1} \in \mathcal{N}(X)$ in the complex case.

Proof. Take a two-dimensional subspace $Y$ of $X$, and write $X=Y \oplus Z$ for suitable $Z$. Now, let $W$ be a two-dimensional space with $n(W)=0\left(\right.$ resp. $n(W)=e^{-1}$ ) in the real (resp. complex) case (see [6] Theorems 3.5 and 3.6]). Then, we have $X \simeq W \oplus_{1} Z$, and [13, Proposition 1] tells us that

$$
n\left(W \oplus_{1} Z\right)=\min \{n(W), n(Z)\}=n(W) .
$$

Our next aim is to prove that $\mathcal{N}(X)$ is an interval for every Banach space $X$. To get it, we will prove the continuity of the mapping carrying every equivalent norm on $X$ to its numerical index with respect to a metric which we explain below. 
The following definitions are taken from [4, 118$]$. We denote by $\mathcal{E}(X)$ the set of all equivalent norms on the Banach space $X$. This is an arcwise connected metric space when provided with the distance given by

$$
d(p, q)=\log (\min \{k \geq 1: p \leq k q, q \leq k p\}) \quad(p, q \in \mathcal{E}(X)) .
$$

If $p \in \mathcal{E}(X)$ and $T \in L(X)$, we write $v_{p}(T)$ for the numerical radius of $T$ in the space $(X, p)$, that is,

$$
v_{p}(T)=\sup \left\{\left|x^{*}(T x)\right|: x \in X, x^{*} \in X^{*}, p(x)=p\left(x^{*}\right)=x^{*}(x)=1\right\},
$$

and $n(X, p)$ will be the numerical index of the Banach space $(X, p)$. Observe that $\mathcal{N}(X)=\{n(X, p): p \in \mathcal{E}(X)\}$.

Proposition 2. Given a Banach space $X$, the mapping $p \longmapsto n(X, p)$ from $\mathcal{E}(X)$ to $\mathbb{R}$ is continuous. Hence, $\mathcal{N}(X)$ is an interval.

Proof. Following the argument in the proof of [4, Theorem 32.4] one can easily show our result, in case $X$ is finite-dimensional. This proof involves a compactness argument which is not available in general, but the ideas to overcome this difficulty can also be found in [4 $\S 18]$. More concretely, an easy refinement in the proof of [4, Corollary 18.4] shows that the mapping

$$
(p, T) \mapsto v_{p}(T) \quad(p \in \mathcal{E}(X), T \in L(X))
$$

is uniformly continuous on bounded sets. Now fix $p_{0} \in \mathcal{E}(X)$, let $B$ be an open ball centered at $p_{0}$ and $S=\left\{T \in L(X): p_{0}(T)=1\right\}$, where we use the same symbol for a norm on $X$ and the associated operator norm. It follows easily from the previous comment and from

$$
\inf \{p(T): p \in B, T \in S\}>0
$$

that the mapping $\Psi: B \times S \rightarrow \mathbb{R}$ given by

$$
\Psi(p, T)=\frac{v_{p}(T)}{p(T)} \quad(p \in B, T \in S)
$$

is uniformly continuous. This implies that the mapping

$$
p \mapsto \inf \{\Psi(p, T): T \in S\}=n(X, p)
$$

is continuous on $B$.

Now we can improve the result in [13] that

$$
\mathcal{N}\left(c_{0}\right)=\mathcal{N}\left(l_{1}\right)=\mathcal{N}\left(l_{\infty}\right)= \begin{cases}{[0,1]} & \text { in the real case } \\ {\left[e^{-1}, 1\right]} & \text { in the complex case }\end{cases}
$$

It follows clearly from Propositions 1 and 2 that the same is true for every Banach space which can be renormed to have numerical index 1 .

Corollary 3. If $1 \in \mathcal{N}(X)$ for a Banach space $X$ of dimension greater than one, then $\mathcal{N}(X)=[0,1]$ in the real case and $\mathcal{N}(X)=\left[e^{-1}, 1\right]$ in the complex case.

Corollary 4 ([19, Theorem 3.2]). Let $m$ be an integer larger than 1 . Then $\mathcal{N}\left(\mathbb{R}^{m}\right)$ $=[0,1]$ and $\mathcal{N}\left(\mathbb{C}^{m}\right)=\left[e^{-1}, 1\right]$. 
In [11, the authors showed that an infinite-dimensional reflexive or quasi-reflexive real Banach space cannot be renormed to have numerical index 1. We will show that this value 1 is very particular. Indeed, "most" Banach spaces can be renormed to have any possible value for the numerical index except eventually 1 . To this end, we set up a relationship between the numerical index and two geometrical properties used in the study of norm attaining operators [10, 17, 18]. Let us recall that a Banach space $X$ has property $(\alpha)$ with constant $\rho(0 \leq \rho<1)$ if there is a family

$$
\left\{\left(x_{i}, x_{i}^{*}\right): i \in I\right\} \subset X \times X^{*}
$$

satisfying

(i) $\left\|x_{i}\right\|=\left\|x_{i}^{*}\right\|=x_{i}^{*}\left(x_{i}\right)=1$ for $i \in I$,

(ii) $\left|x_{i}^{*}\left(x_{j}\right)\right| \leq \rho$ for $i, j \in I, i \neq j$,

(iii) $\alpha\left\|x^{*}\right\|=\sup \left\{\left|x^{*}\left(x_{i}\right)\right|: i \in I\right\}$ for $x^{*} \in X^{*}$.

If the family satisfies (i), (ii), and

(iii) $\beta\|x\|=\sup \left\{\left|x_{i}^{*}(x)\right|: i \in I\right\}$ for $x \in X$,

we say that the Banach space $X$ has property $(\beta)$ with constant $\rho$. More information about properties $(\alpha)$ and $(\beta)$ can be found in [7, 9, 14, 15].

Our next results show that a Banach space has a "large" numerical index whenever it has one of the properties $(\alpha)$ or $(\beta)$ with "small" constant.

Proposition 5. If a Banach space $X$ has property $(\beta)$ with constant $\rho$, then

$$
n(X) \geq \frac{1-\rho}{1+\rho} .
$$

Proof. Let $\left\{\left(x_{i}, x_{i}^{*}\right): i \in I\right\}$ be the family appearing in the definition of property $(\beta)$. We will prove that $v(T) \geq \frac{1-\rho}{1+\rho}\|T\|$ for every $T \in L(X)$. Given $\varepsilon>0$, take $x \in X$ with $\|x\|=1$, such that $\|T x\|>\|T\|-\varepsilon$. Now, use condition (iii) $\beta$ to find $j \in I$ such that

$$
\left|x_{j}^{*}(T x)\right|>\|T\|-\varepsilon \text {. }
$$

Let us write $\left.\left.\lambda=\frac{1-\rho}{1+\rho} \in\right] 0,1\right], t=\frac{1+\lambda x_{j}^{*}(x)}{2} \in[0,1]$, and

$$
z=\lambda x+\left(1-\lambda x_{j}^{*}(x)\right) x_{j}, \quad w=\lambda x-\left(1+\lambda x_{j}^{*}(x)\right) x_{j} .
$$

We clearly have $x_{j}^{*}(z)=1, x_{j}^{*}(w)=-1$, and $\left|x_{i}^{*}(z)\right| \leq 1,\left|x_{i}^{*}(w)\right| \leq 1$ for $i \neq j$, so $\|z\|=\|w\|=1$. Since $\lambda x=t z+(1-t) w$, (1) implies

$$
\left|x_{j}^{*}(T z)\right|>\lambda[\|T\|-\varepsilon] \quad \text { or } \quad\left|x_{j}^{*}(T w)\right|>\lambda[\|T\|-\varepsilon] .
$$

It follows that $v(T) \geq \lambda\|T\|$, as required.

In the complex case this proposition can be improved as follows.

Proposition 6. If a complex Banach space $X$ has property $(\beta)$ with constant $\rho$, then $n(X) \geq 1-\rho$.

Proof. The proof is very close to that given for Proposition [5 so we only mention the changes. We will prove that $v(T) \geq(1-\rho)\|T\|$ for every $T \in L(X)$. Given 
$\varepsilon>0$, take $x \in X$ with $\|x\|=1$ such that $\|T x\|>\|T\|-\varepsilon$, and then use property $(\beta)$ to find $j \in I$ such that, after a suitable rotation,

$$
\operatorname{Re}\left(x_{j}^{*}(T x)\right)=\left|x_{j}^{*}(T x)\right|>\|T\|-\varepsilon .
$$

Now, put $\lambda=1-\rho \in] 0,1]$, and

$$
\mu=\lambda x_{j}^{*}(x)+a i, \quad \nu=\lambda x_{j}^{*}(x)-a i,
$$

where $a \in[0,1]$ is chosen in such a way that $|\mu|=|\nu|=1$. Then define $z, w \in X$ by

$$
z=\lambda x+a i x_{j}, \quad w=\lambda x-a i x_{j},
$$

and continue as in Proposition 5 .

As the dual of a Banach space with property $(\alpha)$ has property $(\beta)$ with the same constant, we get:

Corollary 7. Let $X$ be a Banach space satisfying property $(\alpha)$ with constant $\rho$. Then $n\left(X^{*}\right) \geq \frac{1-\rho}{1+\rho}$ in the real case and $n\left(X^{*}\right) \geq 1-\rho$ in the complex case.

Remark 8. As an easy consequence of the above results, we get that a Banach space satisfying properties $(\alpha)$ or $(\beta)$ with constant $\rho=0$ has numerical index 1 , but actually, these results are not new. On one hand, if a Banach space satisfies property $(\alpha)$ with constant 0 , then it is (isometrically isomorphic to) an $l_{1}(\Gamma)$ space [14, Proposition 2.3]. On the other hand, if it satisfies property $(\beta)$ with constant 0 , then it is (isometrically isomorphic to) a subspace of an $l_{\infty}(\Gamma)$ space containing $c_{0}(\Gamma)$, and in such a case, the space has numerical index 1 by [1, Teorema 5.9].

Using well-known theorems on renorming Banach spaces with these properties, we get the following results.

Theorem 9. Let $X$ be a Banach space of dimension greater than one. Then $\mathcal{N}(X) \supset\left[0,1 / 3\left[\right.\right.$ in the real case and $\mathcal{N}(X) \supset\left[e^{-1}, 1 / 2[\right.$ in the complex case.

Proof. J. Partington [17] proved that every real Banach space can be renormed to have property $(\beta)$, but his proof actually works in the complex case as well. Moreover, by carefully reading the proof, one observes that for every $\varepsilon>0$, the space can be renormed to satisfy property $(\beta)$ with constant less than $1 / 2+\varepsilon$. Now, the result follows from Propositions 1, 2, 5 and 6 ,

Our next results use property $(\alpha)$. Unlike $(\beta)$, property $(\alpha)$ is not isomorphically trivial. Indeed, under the continuum hypothesis there are Banach spaces which cannot be renormed with property $(\alpha)[9,16$. Nevertheless, many Banach spaces can be renormed to satisfy this property, even with arbitrarily small constant $\rho>0$. Therefore, we will improve Theorem 9 for a wide class of Banach spaces. Recall that a system $\left\{\left(x_{\lambda}, x_{\lambda}^{*}\right)\right\}_{\lambda \in \Lambda} \subset X \times X^{*}$ is said to be biorthogonal if $x_{\lambda}^{*}\left(x_{\mu}\right)=\delta_{\lambda, \mu}$ for $\lambda, \mu \in \Lambda$, and long if the cardinality of $\Lambda$ coincides with the density character of $X$.

Theorem 10. Let $X$ be a Banach space admitting a long biorthogonal system. Then $\sup \mathcal{N}(X)=1$. Therefore, $\mathcal{N}(X) \supset\left[0,1\left[\right.\right.$ in the real case and $\mathcal{N}(X) \supset\left[e^{-1}, 1[\right.$ in the complex case. 
Proof. The proof is completely analogous to that given for Theorem 9, using the fact that $X$ admits equivalent norms satisfying property $(\alpha)$ with arbitrarily small constant $\rho>0$ [9, Theorem 1].

Typical examples of Banach spaces admitting a long biorthogonal system are WCG spaces (see [5]).

Infinite-dimensional reflexive or quasi-reflexive real Banach spaces cannot be renormed to have numerical index 1 [1], but they admit long biorthogonal systems. So, for these spaces the inclusion in Theorem 10 is an equality. Actually, if $X^{* *} / X$ is separable, then $X$ is WCG (see, for example, [20, Theorem 3]) and, in the real case, $X$ cannot be renormed to have numerical index 1 [11, Corollary 5]. Therefore, we have

Corollary 11. Let $X$ be an infinite-dimensional real Banach space with $X^{* *} / X$ separable. Then $\mathcal{N}(X)=[0,1[$.

An example of a Banach space which cannot be renormed to have property $(\alpha)$ is a $C(K)$ space, where $K$ is the compact space constructed by K. Kunen (see [16]). Since $n(C(K))=1$, Corollary 3 implies that this space can be renormed with any possible value of the numerical index. We do not know of an example of a Banach space $X$ such that $\sup \mathcal{N}(X)<1$.

\section{ACKNOWLEDGEMENT}

This paper was completed while the first author visited the University of Granada. She thanks the Departamento de Análisis Matemático for their support.

\section{REFERENCES}

1. M. D. Acosta, Operadores que alcanzan su radio numérico, Tesis doctoral, Secretariado de publicaciones, Universidad de Granada. 1990.

2. H. F. Bohnenblust, and S. Karlin, Geometrical properties of the unit sphere in Banach algebras, Ann. of Math. 62 (1955), 217-229. MR 17:177a

3. F. F. Bonsall, and J. Duncan, Numerical Ranges of Operators on Normed Spaces and of Elements of Normed Algebras, London Math. Soc. Lecture Note Series 2, Cambridge 1971. MR 44:5779

4. F. F. Bonsall, and J. Duncan, Numerical Ranges II, London Math. Soc. Lecture Note Series 10, Cambridge 1973. MR 56:1063

5. R. Deville, G. Godefroy, and V. Zizler, Smoothness and Renormings in Banach spaces, Pitman Monographs 64, New York 1993. MR 94d:46012

6. J. Duncan, C. M. McGregor, J. D. Pryce, and A. J. White, The numerical index of a normed space, J. London Math. Soc. (2) 2 (1970), 481-488. MR 41:8967

7. C. Finet, and W. Schachermayer, Equivalent norms on separable Asplund spaces, Studia Math. 92 (1989), 275-283. MR 90d:46026

8. B. W. Glickfeld, On an inequality of Banach algebra geometry and semi-inner-product space theory, Illinois J. Math. 14 (1970), 76-81. MR 40:6239

9. B. V. Godun, and S. L. Troyanski, Renorming Banach spaces with fundamental biorthogonal system, Contemporary Math. 144 (1993), 119-126. MR 94c:46020

10. J. Lindenstrauss, On operators which attain their norm, Israel J. Math. 1 (1968), 139-148. MR 28:3308

11. G. López, M. Martín, and R. Payá, Real Banach spaces with numerical index 1, Bull. London Math. Soc. 31 (1999), 207-212. MR 99k:46024

12. M. Martín, A survey on the numerical index of a Banach space, Extracta Math. 15 (2000), 265-276. MR 2002b:46027

13. M. Martín, and R. Payá, Numerical index of vector-valued function spaces, Studia Math. 142 (2000), 269-280. MR 2001i:46017 
14. J. P. Moreno, On geometry of Banach spaces with property $\alpha$, J. Math. Anal. App. 201 (1996), 600-608. MR 97f:46023

15. J. P. Moreno, Geometry of Banach spaces with $(\alpha, \varepsilon)$-property or $(\beta, \varepsilon)$-property, Rocky Mountain J. Math. 27 (1997), 241-256. MR 99b:46017

16. S. Negrepontis, Banach spaces and topology, in: "Handbook of set theoretic topology" (K. Kunen and J. E. Vaughan, eds.), North Holland, Amsterdam, 1984. MR 86i:46018

17. J. R. Partington, Norm attaining operators, Israel J. Math. 43 (1982), 273-276. MR 85a: 47012

18. W. Schachermayer, Norm attaining operators and renormings of Banach spaces, Israel J. Math 44 (1983), 201-212. MR 84e:46012

19. K. Tillekeratne, Spatial numerical range of an operator, Proc. Camb. Phil. Soc. 76 (1974), 515-520. MR 49:11281

20. M. Valdivia, Topological direct sum decompositions of Banach spaces, Israel J. Math. 71 (1990), 289-296. MR 92d:46047

Institut de Mathématique et D'Informatique, Université de Mons-Hainaut, Avenue DU CHAMP DE MARS 8, B-7000 Mons, BElgium

E-mail address: catherine.finet@umh.ac.be

Departamento de Análisis Matemático, Facultad de Ciencias, Universidad de Granada, 18071 Granada, Spain

E-mail address: mmartins@ugr.es

Departamento de Análisis Matemático, Facultad de Ciencias, Universidad de Granada, 18071 Granada, Spain

E-mail address: rpaya@ugr.es 